\title{
Gestational diabetes, insulin resistance and physical activity in pregnancy in a multi-ethnic population - a public health perspective
}

\author{
Anne Karen Jenum ${ }^{1,2}$, Kåre Rønn Richardsen², Sveinung Berntsen ${ }^{3}$ and Kjersti Mørkrid ${ }^{4,5}$ \\ 1) Faculty of Medicine, University of Oslo, Institute of Health and Society, Department of General Practice \\ 2) Faculty of Health, Oslo and Akershus University College of Applied Sciences \\ 3) Department of Public Health, Sport and Nutrition, Faculty of Health and Sport Sciences, University of Agder \\ 4) Department of Endocrinology, Obesity and Preventive Medicine, Oslo University Hospital \\ 5) Faculty of Medicine, University of Oslo, Institute of Clinical Medicine \\ Correspondence: Anne Karen Jenum, Institute of Health and Society, Department of General Practice, University of Oslo, \\ P.O. Box 1130 Blindern, NO-0318 Oslo, Norway \\ E-mail: a.k.jenum@medisin.uio.no
}

\begin{abstract}
Aims: To summarize findings from the STORK-Groruddalen Study regarding ethnic differences in the prevalence of gestational diabetes (GDM) by the WHO and modified International Association of Diabetes and Pregnancy Study Groups (IADPSG) criteria (no one hour value), insulin resistance, $\beta$-cell function and physical activity (PA) level.

Methods: Population-based cohort study of 823 healthy pregnant women (59\% ethnic minorities). Data from questionnaires, fasting blood samples, anthropometrics and objectively recorded PA level (SenseWear Armband), were collected at $<20$ (Visit 1) and $28 \pm 2$ (Visit 2) weeks of gestation. The 75-g OGTT was performed at Visit 2. Insulin resistance (HOMA-IR) and $\beta$-cell function (HOMA- $\beta$ ) were estimated from venous fasting plasma glucose and C-peptide.

Results: The GDM prevalence was $13.0 \%$ with the WHO and $31.5 \%$ with the IADPSG criteria. The ethnic minority women, especially South Asians, had highest figures. South and East Asian women had highest HOMA-IR at Visit 1 after adjustment for BMI. HOMA-IR increased from Visit 1 to Visit 2 irrespective of ethnic origin. Compared with Western European women, the absolute and percentage increase in HOMA- $\beta$ from Visit 1 to Visit 2 was poorest for the South and East Asian women. All ethnic groups walked less and spent less time in moderate-to-vigorous physical activity (MVPA) during weekend days compared with weekdays. South Asian women were least active, measured by steps and by time spent in MVPA.

Conclusion: Alarmingly high rates of GDM were found, highest among South Asians. South Asian women were less physically active, more insulin resistant and showed poorer $\beta$-cell compensation compared with Western Europeans.
\end{abstract}

\section{INTRODUCTION}

Today, increasing rates of gestational diabetes mellitus (GDM), defined as any degree of glucose intolerance with onset or first recognition during pregnancy, are reported from several continents $(1,2)$. This trend is linked to the global epidemic of obesity, and reflects the underlying prevalence of type 2 diabetes (T2DM) in women of reproductive age in the background population. Comparison of prevalence figures for GDM between study populations is hampered by the lack of consensus about diagnostic criteria and screening procedures (3), in addition to the differences in prepregnancy BMI, age and ethnic origin. The International Association of Diabetes and Pregnancy Study Groups (IADPSG) proposed new criteria for GDM in 2010 (Table 1) (4). These were based on the findings from the Hyperglycaemia and Adverse Pregnancy Outcome (HAPO) study, showing a continuous relationship between maternal glucose levels and adverse fetal outcomes. The IADPSG criteria were set to reflect an odds ratio (OR) of at least 1.75 for neonatal birth weight, cord C-peptide or per cent body fat above the 90th percentile compared with neonates of women with glucose values below the mean. However, other ORs were discussed (4). The IADPSG criteria were adopted by the American Diabetes Association (ADA) in 2011 (5). However, the Carpenter and Coustan criteria have been widely used in North America (6), and the WHO (7) and European Association for the Study of Diabetes (EASD) (8) criteria in Europe (Table 1).

Overweight and obese women start their pregnancy more insulin resistant compared with normal weight women (9). To maintain normoglycaemica during pregnancy, an increase in insulin secretion is needed to compensate for the pregnancy-induced insulin resistance (10). Pregnant women without contraindications are recommended to spend 30 minutes on most, if not all days of the week, in moderate-to-vigorous-intensity physical activity (MVPA) (11). Pregnant women are reported to be less physically active than non-pregnant women both in leisure-time and at work (12). 
Table 1. Gestational diabetes mellitus diagnostic thresholds for 75 -g and 100 -g oral glucose tolerance tests. Values are from venous plasma $(\mathrm{mmol} / \mathrm{l})$.

\begin{tabular}{lcccc}
\hline & \multicolumn{3}{c}{$\begin{array}{c}\text { 75-g oral glucose } \\
\text { tolerance test }\end{array}$} & $\begin{array}{c}\text { 100-g oral glucose } \\
\text { tolerance test }\end{array}$ \\
\cline { 2 - 5 } & $\begin{array}{c}\text { World Health } \\
\text { Organization criteria (7) }\end{array}$ & $\begin{array}{c}\text { European Association } \\
\text { for the Study of } \\
\text { Diabetes (8) }\end{array}$ & $\begin{array}{c}\text { International Association of } \\
\text { Diabetes and Pregnancy } \\
\text { Study Groups (4) }\end{array}$ & $\begin{array}{c}\text { Carpenter and } \\
\text { Coustan (6)* }\end{array}$ \\
\hline Fasting & $\geq 7.0$ & $\geq 6.0$ & $\geq 5.1$ & $\geq 5.3$ \\
$1 \mathrm{~h}$ & & & $\geq 10.0$ & $\geq 10.0$ \\
$2 \mathrm{~h}$ & $\geq 7.8$ & $\geq 9.0$ & $\geq 8.5$ & $\geq 8.6$ \\
$3 \mathrm{~h}$ & & & $\geq 7.8$ \\
\hline
\end{tabular}

*Two values above thresholds necessary for a diagnosis of Gestational diabetes mellitus.

Ethnic minority groups, which are often socially disadvantaged (13), have a higher prevalence of T2DM (14) and GDM (15). Studies from Europe have found that women originating from African and Middle Eastern countries, tend to enter their pregnancies with higher BMI levels than the ethnic majority population in each country $(16,17)$, while women of South and East Asian descent overall were leaner that the European population. However, these findings need to be interpreted in view of studies showing substantial ethnic differences in the amount of body fat relative to BMI, especially in Asians (18). The body fat percentage appears to be from 1-8\% higher among Asians compared with Caucasians at a given BMI, sex and age (19).

The STORK Groruddalen Study was set up in Groruddalen, a part of Oslo, covering affluent as well as more deprived residential areas, with 82000 inhabitants of which about $40 \%$ are ethnic minorities, with a diverse socioeconomic status (20). The majority (75$85 \%$ ) of pregnant women residing in the area attend the Child Health Clinics for antenatal care. In this paper we summarize the main published findings from the STORK Groruddalen Study regarding ethnic differences in 1) the prevalence of GDM by the WHO and modified IADPSG criteria (17), 2) insulin resistance and beta cell function from early pregnancy to 28 weeks of gestation (21) and 3) objectively recorded physical activity (PA) level in early gestation (22). Lastly, we will discuss the public health aspects of GDM.

\section{METHODS}

\section{Design, data collection}

The STORK Groruddalen study is a population-based cohort study, conducted at three public Child Health Clinics in Oslo, Norway, from May 2008 to May 2010 (20). The Regional Ethics committee and The Norwegian Data Inspectorate approved the study protocol. Information material and questionnaires were translated to Arabic, English, Sorani, Somali, Tamil, Turkish, Urdu and Vietnamese. Women were eligible if they 1) lived in the districts, 2) planned to give birth at one of two study hospitals, 3) were less than 20 weeks pregnant, 4) could communicate in Norwegian or any of the above specified languages and 5) were able to give a written consent. Women with pregestational diabetes or other diseases necessitating intensive hospital follow-up during pregnancy, were excluded. In short, questionnaire data, objectively recorded PA, venous fasting blood samples and anthropometrics (body height measured to the nearest $0.1 \mathrm{~cm}$, and body weight to the nearest $0.1 \mathrm{~kg}$ by Tanita-BC $418 \mathrm{MA}$ ) were collected at Visit 1 in early pregnancy (V1, mean; standard deviation (S.D.), gestational week 15.0 (3.4) $(17,20)$. The same data (not background information) were collected at Visit 2 (V2) in weeks $28 \pm 2$ of gestation, when the $75 \mathrm{~g}$ oral glucose tolerance test (OGTT) also was performed.

\section{Main variables}

\section{Ethnicity}

Ethnicity may be defined as the social group a person belongs to, which implies both shared culture, history, geographical origins, language, lifestyle factors, physical, genetic and other factors (23). Ethnic origin was defined by the participant's country of birth or the participant's mother's country of birth if the participant's mother was born outside Europe or North-America (20). Women with ethnic origin from Eastern Europe, Asia, Africa, and South- and Central America are referred to as ethnic minority women, and constituted $59 \%$ of the sample, with the largest groups being South Asian (25\%) and Middle Eastern (15\%). Western Europeans, with the majority born in Norway (93.6\%) or Sweden or Denmark (3.2\%), constitute the reference group.

GDM definitions, insulin resistance and $\beta$-cell function At V2, fasting plasma glucose (FPG) and 2-hour plasma glucose $(\mathrm{PG})$ from the OGTT was measured on site in venous EDTA blood (HemoCue 201+, Angelholm, Sweden) (17). GDM was diagnosed with the WHO criteria: FPG $\geq 7.0$ or 2 -hour $P G \geq 7.8 \mathrm{mmol} / 1$ (7), and modified IADPSG criteria: FPG $\geq 5.1$ or 2 -hour PG $\geq 8.5 \mathrm{mmol} / \mathrm{l}$ (4), as 1 -hour PG values were not available (Table 1).

The homeostatic model assessment (HOMA) was used to estimate insulin resistance (HOMA-IR) and $\beta$ cell function (HOMA- $\beta$ ) at V1 and V2 (21). The Oxford University HOMA Calculator 2.2 was used with FPG and fasting C-peptide concentrations (24). 
Table 2. Baseline characteristics of the total cohort, stratified into Western European and ethnic minority women, and further into ethnic origin. Data are mean (SD), unless otherwise stated.

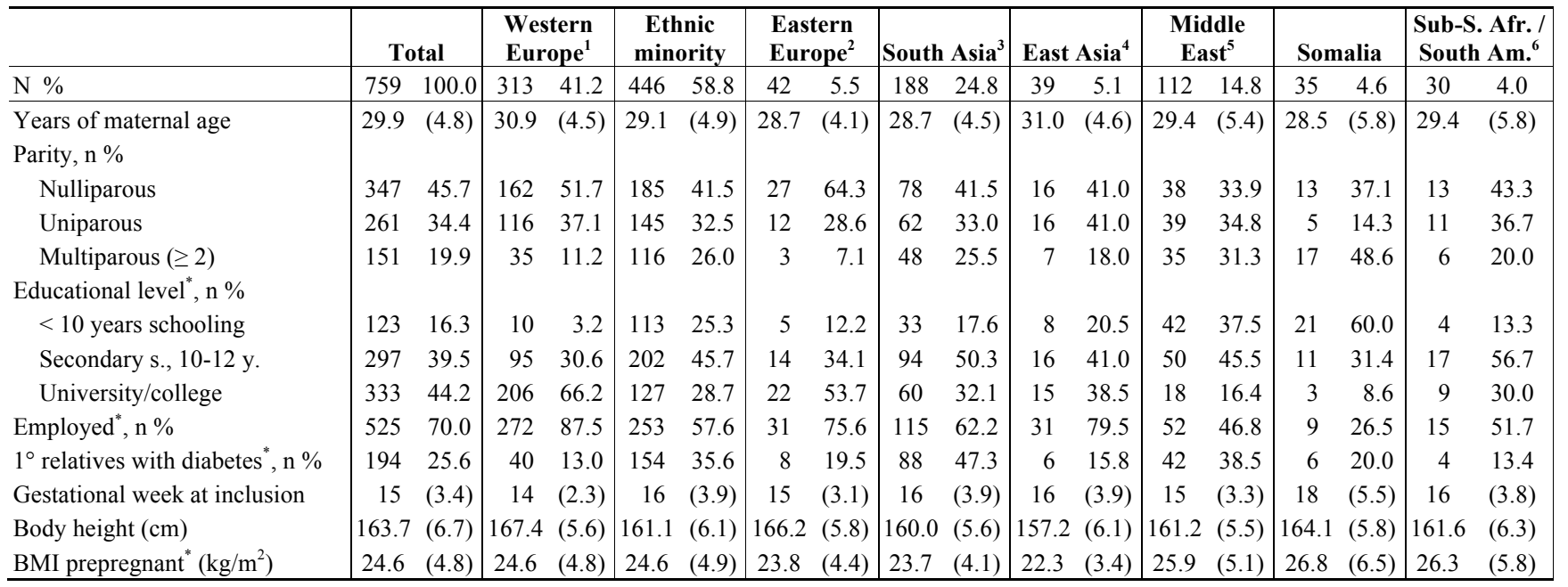

"Incomplete data on the variables because of missing values for 6-19 women.

${ }^{1}$ Norway: 93.6\%, Sweden and Denmark: 3.2\%, other Western Europe (includes North America n=3): $3.2 \%$

${ }^{2}$ Poland: $16.7 \%$, Russia: $14.3 \%$, Kosovo: $14.3 \%$, other Eastern Europe: $54.8 \%$

${ }^{3}$ Pakistan: $62.2 \%$, Sri Lanka: $30.9 \%$, other South Asia: 6.9\%

${ }^{4}$ Vietnam: $43.6 \%$, Philippines: $28.2 \%$, Thailand: 10.3\%, other Eastern Asia: $17.9 \%$

${ }^{5}$ Iraq: $30.4 \%$, Turkey: $22.3 \%$, Marocco: $19.6 \%$, Afghanistan:10.7\%, other Middle East: $16.8 \%$

${ }^{6}$ Sub-Sahara Africa: $53.3 \%$, South/Central America: 33.3\%, Nigeria: $13.3 \%$

For these analyses, FPG was measured from venous blood on gel tubes, analysed at the Department of Multidisciplinary Laboratory Medicine and Medical Biochemistry, Akershus University Hospital (Vitros 5.1 FS, Ortho Clinical Diagnostics, slide adapted colorimetric method). Fasting C-peptide was measured at the Hormone Laboratory, Oslo University Hospital by non-competitive immunofluorometric assays (DELFIA, PerkinElmer Life Sciences, Wallac Oy, Turku, Finland)

Objective recordings of physical activity

PA was objectively recorded at V1 with SenseWear ${ }^{\mathrm{TM}}$ Pro3 Armband (BodyMedia Inc., Pittsburgh, PA, USA) (22), according to protocol and worn the following 4-7 days, only removed for bathing/water activity. Hours of MVPA and steps, per weekday and weekend day, are reported. At least 19.2 hours $(80 \%)$ of one day of Armband use was required to be registered with valid data (22). The cut-off points defining MVPA were 3 metabolic equivalents (METs). The device has been found valid in free living activities (25), and in pregnant women $(26,27)$.

\section{The study sample in the three papers}

In total, 823 ( $74 \%$ of eligible) women were included (20), 772 met at V2, and 759 women with valid OGTT data could be classified according to the GDM criteria (17). These 759 women constitute the study sample for the analyses related to the GDM prevalence. Baseline characteristics are presented in Table 2. No significant differences were found in baseline characteristics between those with and without OGTT data.
There were 695 singleton pregnant women with available FPG and C-peptide values from both V1 and V2 (21). These women constitute the study sample for the analyses related to HOMA figures. No significant differences between those with and without valid data were found with respect to age, parity, prepregnant BMI, body height, level of education or Western European vs. ethnic minority origin.

The 678 women with valid Armband data constitute the study sample for the analysis related to objectively recorded PA at V1 (22). Mean age, weeks of gestation at inclusion, body mass, height and BMI did not differ between women with valid PA recordings and those without.

\section{Statistical analyses}

Simple descriptive tests were used as appropriate. Bonferroni-corrections for multiple testing were done. Multiple logistic regression analyses were performed to identify the effect of ethnic origin on GDM with the WHO and modified IADPSG criteria separately. Age, prepregnant BMI and parity were entered as adjustment covariates in model $\mathrm{A}$, thereafter education and body height (used as a proxy for early life socioeconomic status) in model $\mathrm{B}$, and lastly first degree relatives with diabetes in model $\mathrm{C}$. Interactions were tested. ORs with 95\% CI were estimated. Multiple linear regression analyses were performed with HOMA-IR at V1 and percentage change in HOMA- $\beta$ from V1 to V2 as dependent variables. Adjustments were done for gestational week and ethnic origin, and further for prepregnant BMI. The residuals were 
checked for normality and independence. SPSS (Statistical Software package) versions 18 and 19 were used in the analyses for these two papers.

Regarding the paper on PA, multivariate linear regression models using robust regression were applied, with hours of MVPA or steps per weekday or weekend day as dependent variables, with BMI, ethnic origin, education, occupation, age, gestational week and parity as independent variables (core set variables). All multivariate models included adjustments for hours and days with PA recordings. The final main-effects model was tested for interactions between ethnicity and parity, education and occupation. We also checked for interactions between parity and BMI. Statistical analyses related to PA were performed with Number Cruncher Statistical System 2007 Version 07.1.12 (NCSS, LLC, Kaysville, Utah, USA). For all analyses the statistical significance level was set to $5 \%$.

\section{RESULTS}

\section{Baseline characteristics}

The mean (S.D.) maternal age was 29.9 years (4.9) and the prepregnant BMI was $24.5 \mathrm{~kg} / \mathrm{m}^{2}$ (4.8) (Table 2). Western European women were older, taller, had lower parity and higher education compared to the ethnic minority women. Mean prepregnant BMI did not differ between Western European and ethnic minority women. Heterogeneity between the ethnic minority groups was observed for several variables such as prepregnant BMI, parity and education.

\section{GDM by the WHO and IADPSG criteria}

The GDM prevalence with the WHO criteria were $13.0 \%$ overall, $10.9 \%$ in Western European and 14.6\% in ethnic minority women, and increased to $31.5 \%$, $24.0 \%$ and $36.8 \%$ respectively, with the modified IADPSG criteria (Figure 1). The increase was strongest in South Asian women (2.8 times, compared with 2.2 times in Western European and Middle Eastern women). FPG $\geq 5.1 \mathrm{mmol} / 1$ was found in $37.2 \%$ of South Asian, $35.7 \%$ of Middle Eastern and $21.4 \%$ of Western European women.

For GDM with the WHO criteria, ethnic minority origin was an independent predictor (South Asians: OR (95\% CI); 2.24 (1.26-3.97), Middle Eastern women: 2.13 (1.12-4.08) adjusted for age, prepregnant BMI and parity (Model A) (Table 3). However, when education and body height (a proxy for early life socioeconomic status) were added (Model B), the increased OR for ethnic minority women was eliminated. Similar results were found in the final model (Model C) with additional adjustments for a family history of diabetes. A different pattern was found when assessing predictors for GDM with the modified IADPSG criteria. All models revealed a significantly increased OR for GDM for ethnic minority women, and adjustment for education, body height and family history of diabetes had little impact (Model B and C).

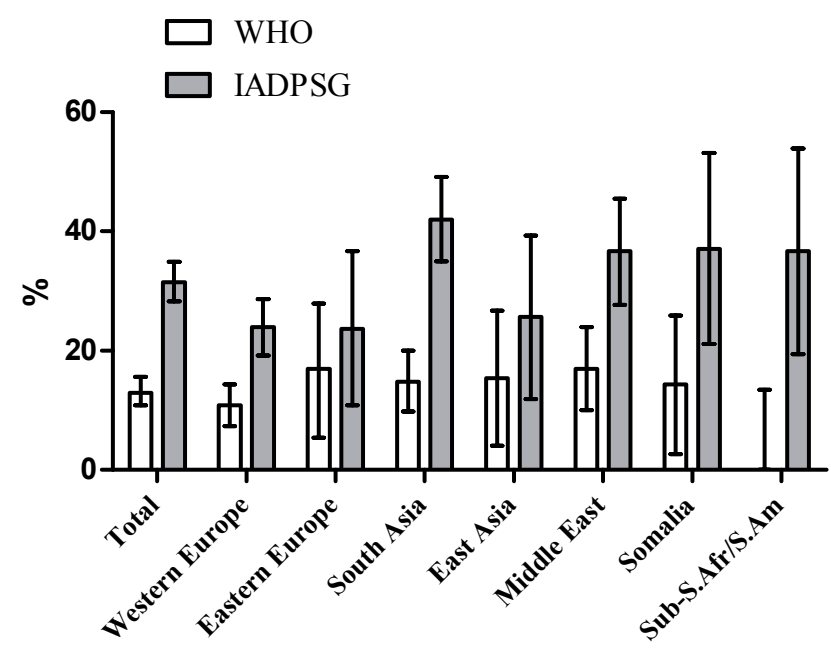

Figure 1. Gestational diabetes prevalence with the WHO (white) and modified IADPSG criteria (grey), for the total cohort and the main ethnic minority groups, based on universal screening with oral glucose tolerance test at $\mathrm{Vi}$ sit 2 (Gestational week $28 \pm 2$ ). Crude values, percentage (95\% CI).

\section{Insulin resistance and beta cell function}

The overall insulin resistance measured by HOMA-IR increased from median (IQR) $1.1(0.6)$ at V1 to 1.7 (0.9) at V2 $(\mathrm{P}<0.001)$. South Asians had higher HOMA-IR compared with Western Europeans at both visits (Figure 2a). No significant ethnic differences were found in the absolute or percentage change in HOMA-IR from V1 to V2. The overall $\beta$-cell function measured by HOMA- $\beta$ increased from V1 to V2 $(p<0.001)$. South Asians had higher HOMA- $\beta$ compared with Western Europeans at V1, but not at V2 (Figure 2b). Compared with Western European women, the absolute and percentage increase in HOMA- $\beta$ from V1 to V2 were less for the South Asian $(p<0.005)$ and East Asian $(\mathrm{p}<0.002)$ women.

In linear regression analyses with HOMA-IR at V1 as the dependent variable, South Asian and Middle Eastern women were more insulin resistant compared with Western Europeans after adjustments for gestational week at inclusion. After further adjustments for prepregnant BMI, South Asians were still more insulin resistant, East Asians became more insulin resistant, while the Middle Easterners were no longer significantly different from the Western Europeans. In analyses with the percentage change in HOMA- $\beta$ from V1 to $\mathrm{V} 2$ as the dependent variable, the increase in $\beta$-cell function in East Asian and South Asian was significantly less than in the Western European women after adjustments for gestational week at inclusion, and further for prepregnant BMI.

\section{Objectively recorded physical activity in early gestation}

Western European women walked more steps compared with South Asian women per weekday and during weekends $(\mathrm{p}<0.001$ for both) (Figure 3a). South Asian women walked less than women from the Middle 
Table 3. Multiple logistic regression models for the gestational diabetes groups identified with the WHO and the modified IADPSG criteria, showing the impact of ethnic origin on gestational diabetes after adjusting for covariates.

\begin{tabular}{|c|c|c|c|c|c|c|c|c|c|c|c|c|}
\hline \multirow{3}{*}{ WHO $n=99$} & \multicolumn{4}{|c|}{ Multiple model A } & \multicolumn{4}{|c|}{ Multiple model B } & \multicolumn{4}{|c|}{ Multiple model C } \\
\hline & OR & 959 & $6 C I$ & $P$ & $O R$ & $95 \%$ & ${ }_{\circ} C I$ & $P$ & OR & $95^{\circ}$ & o $C I$ & $P$ \\
\hline & 1.13 & 1.08 & 1.19 & $<0.00$ & 1.13 & 1.08 & 1.19 & $<0.00$ & 1.12 & 1.06 & 1.18 & $<0.00$ \\
\hline Body height & & & & & 0.93 & 0.89 & 0.97 & $<0.00$ & 0.92 & 0.88 & 0.96 & $<0.00$ \\
\hline BMI prepregnant & 1.05 & 1.00 & 1.09 & 0.044 & 1.04 & 1.00 & 1.09 & 0.054 & 1.04 & 0.99 & 1.09 & 0.111 \\
\hline Parous $\geq 1$ & 2.08 & 1.29 & 3.36 & 0.003 & 2.29 & 1.39 & 3.76 & 0.001 & 2.33 & 1.39 & 3.90 & 0.001 \\
\hline$<10$ years education & & & & & 1.91 & 1.05 & 3.47 & 0.034 & 1.88 & 1.01 & 3.49 & 0.047 \\
\hline $1^{\circ}$ relatives with diabetes & & & & & & & & & 1.89 & 1.32 & 2.71 & 0.001 \\
\hline Ethnic origin ref. Western Europe & & & & 0.026 & & & & 0.772 & & & & 0.771 \\
\hline Sou & 2.24 & 1.26 & 3.97 & 0.006 & 1.18 & 0.62 & 2.27 & 0.614 & 0.76 & 0.38 & 1.55 & 0.456 \\
\hline Mido & 2.13 & 1.12 & 4.08 & 0.022 & 1.03 & 0.49 & 2.16 & 0.944 & 0.77 & 0.35 & 1.66 & 0.503 \\
\hline Other $\mathrm{m}$ & 1.45 & 0.77 & 2.73 & 0.244 & 0.82 & 0.41 & 1.65 & 0.580 & 0.69 & 0.33 & 1.43 & 0.314 \\
\hline$I A D P S G n=$ & $O R$ & 959 & $6 C I$ & $P$ & $O R$ & $95 \%$ & o $C I$ & $P$ & $O R$ & $95^{\circ}$ & oCI & $P$ \\
\hline Years of ma & 1.03 & 1.00 & 1.07 & 0.068 & 1.04 & 1.00 & 1.08 & 0.054 & 1.03 & 0.99 & 1.07 & 0.116 \\
\hline Body he & & & & & 1.01 & 0.98 & 1.03 & 0.641 & 1.00 & 0.98 & 1.03 & 0.746 \\
\hline BMI prepregn & 1.10 & 1.06 & 1.13 & $<0.00$ & 1.09 & 1.06 & 1.13 & $<0.00$ & 1.09 & 1.05 & 1.13 & $<0.00$ \\
\hline Parous $\geq 1$ & 1.17 & 0.82 & 1.65 & 0.390 & 1.21 & 0.85 & 1.73 & 0.281 & 1.15 & 0.81 & 1.64 & 0.438 \\
\hline$<10$ years education & & & & & 1.50 & 0.96 & 2.36 & 0.078 & 1.55 & 0.98 & 2.45 & 0.062 \\
\hline $1^{\circ}$ relatives with diabetes & & & & & & & & & 1.10 & 0.83 & 1.47 & 0.510 \\
\hline Ethnic origin ref. Western Europe & & & & $<0.00$ & & & & $<0.00$ & & & & 0.002 \\
\hline South Asia & 2.94 & 1.94 & 4.47 & $<0.00$ & 2.93 & 1.84 & 4.67 & $<0.00$ & 2.54 & 1.56 & 4.13 & $<0.00$ \\
\hline Middle East & 1.79 & 1.10 & 2.93 & 0.019 & 1.58 & 0.92 & 2.73 & 0.100 & 1.44 & 0.83 & 2.51 & 0.196 \\
\hline Other minorities ${ }^{*}$ & 1.44 & 0.91 & 2.28 & 0.120 & 1.35 & 0.82 & 2.21 & 0.234 & 1.25 & 0.76 & 2.06 & 0.381 \\
\hline
\end{tabular}

*includes Eastern Europe, East Asia, Somalia and Sub Saharan Africa/South America.

a)

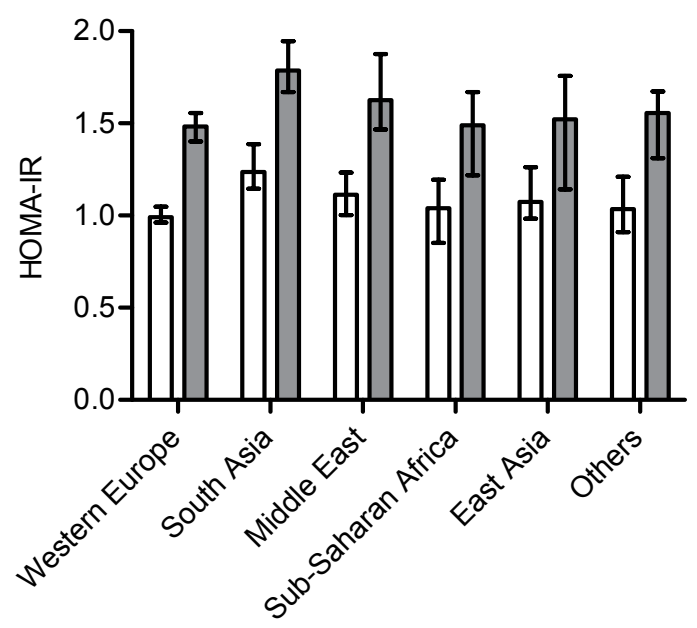

b)

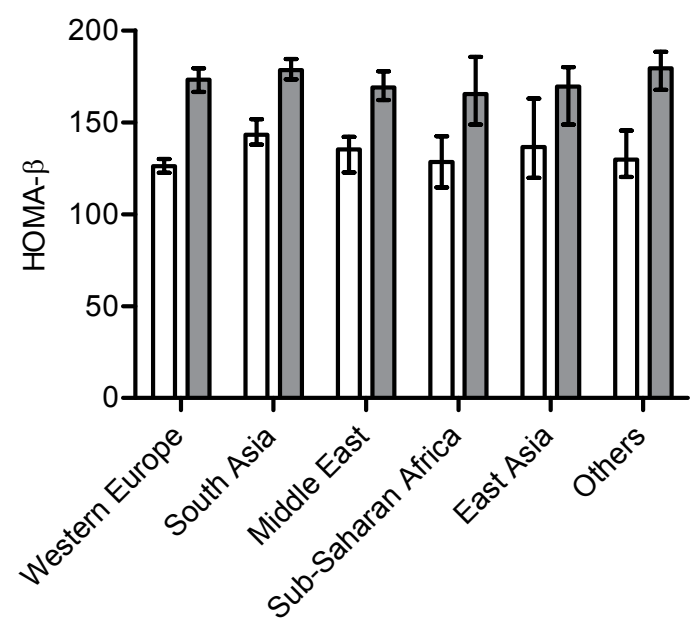

Figure 2a) Insulin resistance assessed by HOMA-IR, and b) $\beta$-cell function assessed by HOMA- $\beta$ for the ethnic groups at Visit 1 (white) and Visit 2 (Gestational week $28 \pm 2$ ) (grey). Crude values, median (95\% CI).

East $(\mathrm{p}=0.004)$ and from other minorities $(\mathrm{p}=0.03)$ during weekdays, while no significant differences were observed between Western European, Middle Eastern or women from other minorities. All ethnic groups walked significantly less $(\mathrm{p}<0.001)$ during weekend days compared with weekdays (Figure 3b).

In the multivariate linear regression model, the number of steps at weekdays and weekend days for South
Asians was lower compared with Western European women, after adjustment for education, employment, age, gestational week, parity, BMI and time wearing the activity monitor. A significant interaction term $(p=0.008)$ between ethnicity (Western European vs. South Asian) and parity was identified. Having one or more children was positively associated with steps during weekends in South Asians, while Western Eu- 

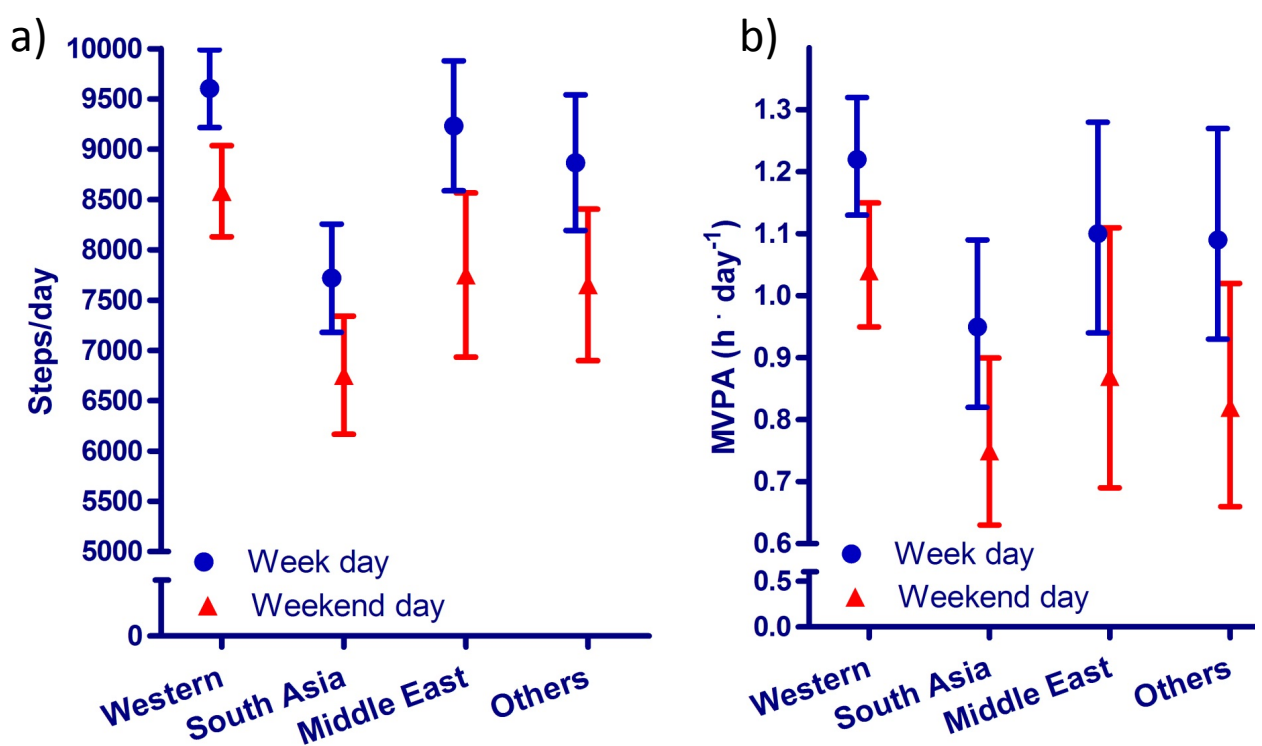

Figure 3a) Steps per week- and weekend day for ethnic groups (mean and 95\% CI), and b) hours per week- and weekend day of moderate physical activity for ethnic groups (geometric mean and 95\% CI). Crude values from Visit 1.

ropean women walked more steps when nulliparous. Furthermore, South Asian women spent less time in MVPA at weekdays and weekend days compared with Western European women after adjustment for core set variables. A significant interaction term for ethnicity and educational level was also found when both steps and MVPA at weekend days were the dependent variables. High education was positively associated with steps and MVPA during weekends in Western European but not in South Asian women.

\section{DisCUSSION}

To our knowledge, the STORK Groruddalen population-based study is the first to compare the prevalence of GDM by the WHO and the IADPSG criteria, assess insulin resistance and $\beta$-cell function and objectively record PA levels in pregnant women with diverse ethnic origin. Based on universal OGTT screening, the prevalence of GDM with the WHO criteria was $11 \%$ in Western European and $15 \%$ in ethnic minority women, but increased to $24 \%$ and $37 \%$ respectively with the modified IADPSG criteria. In early gestation, East and South Asian women were more insulin resistant compared with Western Europeans. Women from all ethnic groups, irrespective of baseline levels, became equally more insulin resistant from early gestation to 28 weeks of gestation, but the $\beta$-cell function of East and South Asians did not compensate to the same extent as in the Western Europeans. Furthermore, South Asian women walked less and spent less time in MVPA than Western European women, and parity and educational level influenced both measures of PA during weekend days differently in these ethnic groups.

The prevalence of GDM by the WHO criteria was higher than suspected in Western European women, and higher than in most $(28)$, but not all $(29,30)$ studies of similar populations. GDM prevalence figures reported from Europe range from 1 to $22 \%$ (3). Observed differences may be attributed to ethnic origin of the populations studied, secular trends for obesity and maternal age, and not least the preference for selective screening with lower sensitivity in most studies $(3,31)$. The prevalence of GDM with the modified IADPSG criteria in our study was higher than in the HAPO study (18\%, range 9.3-25.5\%). (32). Similar high rates as we found in the ethnic minorities, have been reported in other high risk ethnic groups (38\%) (33), which may be underrepresented in the HAPO cohort.

In line with other studies $(34,35)$, the Middle Eastern women in our study had a higher mean BMI, which explained their higher insulin resistance. Our finding that East and South Asian women became more insulin resistant after adjustments for BMI is in accordance with other studies $(36,37)$, and supported by findings that Asians have more fat per BMI unit compared with Western subjects (19). Regardless of ethnic origin and baseline level, the insulin resistance increased approximately $40-45 \%$ from early pregnancy to 28 weeks of gestation, in line with previous reports (9). However, the $\beta$-cell response observed for women from East and South Asia was less than in their Western European counterparts. The $\beta$-cell response in relation to the pregnancy induced insulin resistance was more adequate for the East Asians compared with South Asians. Furthermore, the East Asians had higher 2-hour PG values than the South Asians (17). This is in line with the HAPO study, reporting that East Asian women were less likely to have a GDM diagnosis based on the FPG value compared with women at other HAPO study sites (32).

We did not identify other studies from Europe exploring ethnic differences in PA level in pregnancy based on objective recordings, but one study from the 
US assessed MVPA levels and found that ethnic minority women (African American, Hispanics or Pacific Islanders) were less likely than white American women to engage in PA (38). Also from studies based on selfreported PA, pregnant White American women are more likely to engage in PA compared with ethnic minority women in the US $(39,40)$. Others have reported no statistically significant association between ethnicity and PA $(41,42)$, which concur with our findings related to the other ethnic groups (Middle Eastern and Other background). Our findings of substantially lower levels of steps and MVPA in weekdays and weekend days in South Asian women compared with other ethnic minority and Western women, are also in line with several studies, mostly based on self-reports, from non-pregnant women (43). This may partly be related to cultural factors, transmitted over generations.

Strengths of our study are the population-based design and the multi-ethnic cohort including recently immigrated and illiterate women often excluded from research, the high attendance rate and minimal loss to follow-up at V2, objectively recorded PA and universal OGTT screening $(17,21,22)$. The study sample was found fairly representative for the largest ethnic groups $(17,20)$. The results should be relevant outside the Norwegian context as similar ethnic minority groups live in many countries. Limitations are dealt with in detail in the respective papers $(17,21,22)$. Obviously, small numbers in some ethnic minority groups limits the power in some analyses. As we did not measure 1-h PG, the IADPSG prevalence might be underestimated. The HOMA is a surrogate measure of insulin resistance and $\beta$-cell function (44), but is feasible in large studies and has been validated in pregnant women (24, 45). Although objectively recorded PA is less prone to underestimation due to recall and social desirability bias, wearing the Armband may be a motivational factor resulting in overestimation of habitual activity.

\section{Implications for public health}

Pregnancy complications like GDM seem to be early markers of disturbances in glucose metabolism (10), endothelial dysfunction and hypertension (46), which in turn, predicts future risk of T2DM in the mother. Women with previous GDM may have a 7-fold risk of future T2DM, compared with normal pregnancies (47). However, T2DM may be prevented or postponed in these women by moderate lifestyle changes (48).

Until now, a 2-step screening procedure has mostly been used in clinical practice and research, implying that only women with specified risk factors have had an oral glucose tolerance test, which is the gold standard for a definite diagnosis of GDM. The IADPSG has proposed universal screening in gestational week 24-28 (4). The lower diagnostic FPG threshold as suggested by the IADPSG, will have a major effect on the numbers diagnosed $(32,49)$. Concerns related to the implications of the IADPSG criteria are being addressed, such as potentially negative consequences of labelling a large number of women (some at relatively low risks) based on a test with poor reproducibility $(49,50)$, the increased work load (51) and the questionable cost-effectiveness of interventions in late pregnancy (50). Although positive short-time effects are observed $(52,53)$, only a marginal effect on the prevalence of macrosomia can be expected (50).

Prepregnant obesity, increasing maternal age and family history of diabetes are established risk factors for GDM (28). Ethnic minority women tend to get GDM at a younger age and a lower BMI than Western European women (54). The role of PA in relation to GDM is not clear, although higher levels of PA prior to and in early gestation are associated with a protective effect (55). The lack of knowledge is probably partly due to the poor performance of self-reported PA, when assessing the dose-response relationship between PA levels and GDM. Our data are based on objective recordings of $\mathrm{PA}$, and we will explore the relationship between PA and GDM in more detail. Socioeconomic status and early life factors, often measured with body height, are inversely associated with GDM in several studies (28).

There is now strong evidence supporting that early development plays a central role in determining an individual's risk of later adult disease (56), by mechanisms of developmental plasticity, including epigenetic processes enabling the development of a phenotype appropriate for the environment in which the offspring is predicted to live (57). If there is a mismatch between this environment and the actual environment the offspring is born into, increased susceptibility to obesity, T2DM and cardiovascular disease might develop (58). In line with this perspective, we found that ethnic differences in OR for GDM by the WHO criteria disappeared after adjusting for relevant covariates and socioeconomic factors. The results were slightly different for GDM by the IADPSG criteria, which so far seem to be driven more by adiposity itself. Both maternal GDM and obesity are independently associated with increased birth weight in the offspring (59). This may create a "vicious cycle" where the increasing prevalence of obesity and T2DM in the mothers endorses obesity (60) and related risks factors for noncommunicable diseases in later generations (56). These findings, and the developmental origin of health and disease concept, support that new public health initiatives need to focus more on early life interventions, as interventions in adulthood have shown limited results (57). Follow-up data of offspring from intervention trials for women with GDM are so far not convincing (61). This may indicate that the window of opportunity to prevent long term predisposition to obesity may be earlier in fetal life than the currently recommended time of screening for GDM. Only population-based approaches to improve living conditions and promote a healthy lifestyle, may effectively modify the trends for the global obesity and T2DM epidemic (62), not least in the most susceptible ethnic groups (33). 


\section{Conclusion}

Alarmingly high rates of GDM were found, not least in ethnic minority women. The prevalence increased 2.4 timers when applying the IADPSG criteria. South Asian women were most insulin resistant and least physically active in early pregnancy, and had the poorest $\beta$-cell compensation from early pregnancy to gestational week 28 and had the highest rates of GDM. Findings from the present study may indicate that South Asian women without children and with higher education are particularly prone to an inactive lifestyle during pregnancy.

\section{ACKNOWLEDGEMENTS}

The Research Council of Norway funded PhD fellowship for $\mathrm{KM}$ and the data collection, also supported by The SouthEastern Norway Regional Health Authority and The Norwegian Directorate of Health. The STORK Groruddalen study originated from Oslo University Hospital, Aker. Close collaborative partners were the City of Oslo (Stovner, Grorud and Bjerke Districts) and their administrative leaders, Akershus University Hospital and Oslo University Hospital, Ullevål. Translation of questionnaires was performed by the City Services Department, The Interpreting and Translating Service in Oslo. We thank H. S. Hatlehol and the other staff at the Child Health Clinics for collecting the data, and $\mathrm{H}$. Skaaland, Oslo University Hospital, Aker, for organizing the database. Not least we thank the participating women.

\section{AUTHOR CONTRIBUTION STATEMENT}

AKJ provided overall leadership and guidance on the development of the paper, in close collaboration with KM related to the papers on GDM, insulin resistance and $\beta$-cell function and $\mathrm{SB}$ and KRR related to the paper about PA. AKJ prepared the first and the revised drafts of the paper, with major contributions from KM, SB and KRR. All authors have read and approved the final version. The authors alone are responsible for the views expressed in the paper.

\section{REFERENCES}

1. Ferrara A. Increasing prevalence of gestational diabetes mellitus: a public health perspective. Diabetes Care 2007; 30 (Suppl 2): S141-6.

2. Anna V, van der Ploeg HP, Cheung NW, Huxley RR, Bauman AE. Sociodemographic correlates of the increasing trend in prevalence of gestational diabetes mellitus in a large population of women between 1995 and 2005. Diabetes Care 2008; 31 (12): 2288-93.

3. Buckley BS, Harreiter J, Damm P, et al. Gestational diabetes mellitus in Europe: prevalence, current screening practice and barriers to screening. Diabet Med 2012; 29: 844-54.

4. Metzger BE, Gabbe SG, Persson B, et al. International association of diabetes and pregnancy study groups recommendations on the diagnosis and classification of hyperglycemia in pregnancy. Diabetes Care 2010; 33 (3): $676-82$.

5. Standards of medical care in diabetes - 2012. Diabetes Care 2012; 35 (Suppl 1): S11-63.

6. Committee opinion no. 504: screening and diagnosis of gestational diabetes mellitus. Obstet Gynecol 2011; 118 (3): 751-3.

7. Alberti KG, Zimmet PZ. Definition, diagnosis and classification of diabetes mellitus and its complications. Part 1: diagnosis and classification of diabetes mellitus provisional report of a WHO consultation. Diabet Med 1998; 15 (7): 539-53.

8. Brown CJ, Dawson A, Dodds R, et al. Report of the Pregnancy and Neonatal Care Group. Diabet Med 1996; 13 (9 Suppl 4): S43-53.

9. Catalano PM. Obesity, insulin resistance, and pregnancy outcome. Reproduction 2010; 140 (3): 365-71.

10. Buchanan TA, Xiang AH, Page KA. Gestational diabetes mellitus: risks and management during and after pregnancy. Nat Rev Endocrinol 2012; 8 (11): 639-49.

11. Exercise during pregnancy and the postpartum period. Clin Obstet Gynecol 2003; 46 (2): 496-9.

12. Gaston A, Cramp A. Exercise during pregnancy: a review of patterns and determinants. J Sci Med Sport 2011; 14 (4): 299-305.

13. Davey Smith G. Learning to live with complexity: ethnicity, socioeconomic position, and health in Britain and the United States. Am J Public Health 2000; 90 (11): 1694-8.

14. Jenum AK, Holme I, Graff-Iversen S, Birkeland K. Ethnicity and sex are strong determinants of diabetes in an urban Western society: implications for prevention. Diabetologia 2005; 48: 435-9.

15. Petry CJ. Gestational diabetes: risk factors and recent advances in its genetics and treatment. Br J Nutr 2010; 104 (6): 775-87.

16. Heslehurst N, Rankin J, Wilkinson JR, Summerbell CD. A nationally representative study of maternal obesity in England, UK: trends in incidence and demographic inequalities in 619323 births, 1989-2007. Int J Obes 2010; 34 (3): 420-8.

17. Jenum AK, Morkrid K, Sletner L, et al. Impact of ethnicity on gestational diabetes identified with the WHO and the modified International Association of Diabetes and Pregnancy Study Groups criteria: a populationbased cohort study. Eur J Endocrinol 2012; 166 (2): 317-24. 
18. WHO IAftSoO, International Obesity TaskForce. The Asia-Pacific Perspective: Redefining Obesity and its Treatment Health Communications. 2000. doi: http: //www.iotf.org/asiapacific/

19. Wulan SN, Westerterp KR, Plasqui G. Ethnic differences in body composition and the associated metabolic profile: a comparative study between Asians and Caucasians. Maturitas 2010; 65 (4): 315-9.

20. Jenum AK, Sletner L, Voldner N, et al. The STORK Groruddalen research programme: A population-based cohort study of gestational diabetes, physical activity, and obesity in pregnancy in a multiethnic population. Rationale, methods, study population, and participation rates. Scand J Public Health 2010; 38 (5 Suppl): 60-70.

21. Morkrid K, Jenum AK, Sletner L, et al. Failure to increase insulin secretory capacity during pregnancyinduced insulin resistance is associated with ethnicity and gestational diabetes. Eur J Endocrinol 2012; 167 (4): 579-88.

22. Berntsen S, Richardsen KR, Morkrid K, Sletner L, Birkeland KI, Jenum AK. Objectively recorded physical activity in early pregnancy: A multiethnic population-based study. Scand J Med Sci Sports 2012 [Epub ahead of print]. doi: 10.1111/sms.12034

23. Bhopal RS. Ethnicity, race, and health in multicultural societies: foundations for better epidemiology, public health and health care. Oxford: Oxford University Press, 2007.

24. Wallace TM, Levy JC, Matthews DR. Use and abuse of HOMA modeling. Diabetes Care 2004; 27 (6): 1487 95 .

25. Berntsen S, Hageberg R, Aandstad A, et al. Validity of physical activity monitors in adults participating in free-living activities. Br J Sports Med 2010; 44 (9): 657-64.

26. Berntsen S, Stafne SN, Morkved S. Physical activity monitor for recording energy expenditure in pregnancy. Acta Obstet Gynecol Scand 2011; 90 (8): 903-7.

27. Smith KM, Lanningham-Foster LM, Welk GJ, Campbell CG. Validity of the SenseWear (R) Armband to predict energy expenditure in pregnant women. Med Sci Sports Exerc 2012; 44 (10): 2001-8.

28. Galtier F. Definition, epidemiology, risk factors. Diabetes Metab 2010; 36: 628-51.

29. Lamberg S, Raitanen J, Rissanen P, Luoto R. Prevalence and regional differences of gestational diabetes mellitus and oral glucose tolerance tests in Finland. Eur J Public Health 2010; 22 (2): 278-80.

30. McCance DR. Gestational Diabetes Mellitus. In: Williams R, Herman W, Kinmonth AL, Wareham N, editors. The Evidence Base for Diabetes Care, 2002: 245-84.

31. Jiwani A, Marseille E, Lohse N, Damm P, Hod M, Kahn JG. Gestational diabetes mellitus: results from a survey of country prevalence and practices. J Matern Fetal Neonatal Med 2012; 25 (6): 600-10.

32. Sacks DA, Hadden DR, Maresh M, et al. Frequency of Gestational Diabetes Mellitus at Collaborating Centers Based on IADPSG Consensus Panel-Recommended Criteria: The Hyperglycemia and Adverse Pregnancy Outcome (HAPO) Study. Diabetes Care 2012; 35 (3): 526-8.

33. Agarwal MM, Dhatt GS, Shah SM. Gestational diabetes mellitus: simplifying the international association of diabetes and pregnancy diagnostic algorithm using fasting plasma glucose. Diabetes Care 2010; 33 (9): 2018 20 .

34. Jenum AK, Diep LM, Holmboe-Ottesen G, Holme IM, Kumar BN, Birkeland KI. Diabetes susceptibility in ethnic minority groups from Turkey, Vietnam, Sri Lanka and Pakistan compared with Norwegians - the association with adiposity is strongest for ethnic minority women. BMC Public Health 2012; 12 (1): 150.

35. Wong VW. Gestational diabetes mellitus in five ethnic groups: a comparison of their clinical characteristics. Diabet Med 2012; 29 (3): 366-71.

36. Eapen D, Kalra GL, Merchant N, Arora A, Khan BV. Metabolic syndrome and cardiovascular disease in South Asians. Vasc Health Risk Manag 2009; 5: 731-43.

37. Misra A, Khurana L. Obesity-related non-communicable diseases: South Asians vs White Caucasians. Int $J$ Obes $2011 ; 35$ (2): 167-87.

38. Evenson KR, Wen F. Prevalence and correlates of objectively measured physical activity and sedentary behavior among US pregnant women. Prev Med 2011; 53 (1-2): 39-43.

39. Ning Y, Williams MA, Dempsey JC, Sorensen TK, Frederick IO, Luthy DA. Correlates of recreational physical activity in early pregnancy. J Matern Fetal Neonatal Med 2003; 13 (6): 385-93.

40. Petersen AM, Leet TL, Brownson RC. Correlates of physical activity among pregnant women in the United States. Med Sci Sports Exerc 2005; 37 (10): 1748-53.

41. Evenson KR, Savitz DA, Huston SL. Leisure-time physical activity among pregnant women in the US. Paediatr Perinat Epidemiol 2004; 18 (6): 400-7.

42. Pereira MA, Rifas-Shiman SL, Kleinman KP, Rich-Edwards JW, Peterson KE, Gillman MW. Predictors of change in physical activity during and after pregnancy: Project Viva. Am J Prev Med 2007; 32 (4): 312-9.

43. Bhopal RS. A four-stage model explaining the higher risk of Type 2 diabetes mellitus in South Asians compared with European populations. Diabet Med 2013; 30 (1): 35-42. 
44. Matthews DR, Hosker JP, Rudenski AS, Naylor BA, Treacher DF, Turner RC. Homeostasis model assessment: insulin resistance and beta-cell function from fasting plasma glucose and insulin concentrations in man. Diabetologia 1985; 28 (7): 412-9.

45. Wallace TM, Matthews DR. The assessment of insulin resistance in man. Diabet Med 2002; 19 (7): $527-34$.

46. Pina IL. Cardiovascular disease in women: challenge of the middle years. Cardiol Rev 2011; 19 (2): $71-5$.

47. Bellamy L, Casas J-P, Hingorani AD, Williams D. Type 2 diabetes mellitus after gestational diabetes: a systematic review and meta-analysis. Lancet 2009; 373 (9677): 1773-9.

48. Ratner RE, Christophi CA, Metzger BE, et al. Prevention of Diabetes in Women with a History of Gestational Diabetes: Effects of Metformin and Lifestyle Interventions. J Clin Endocrinol Metab 2008; 93 (12): 4774-9.

49. Holt RI, Coleman MA, McCance DR. The implications of the new International Association of Diabetes and Pregnancy Study Groups (IADPSG) diagnostic criteria for gestational diabetes. Diabet Med 2011; 28 (4): 382-5.

50. Ryan EA. Diagnosing gestational diabetes. Diabetologia 2011; 54 (3): 480-6.

51. Moses RG. New consensus criteria for GDM: problem solved or a pandora's box? Diabetes Care 2010; 33 (3): 690-1.

52. Crowther CA, Hiller JE, Moss JR, McPhee AJ, Jeffries WS, Robinson JS. Effect of treatment of gestational diabetes mellitus on pregnancy outcomes. $N$ Engl J Med 2005; 352 (24): 2477-86.

53. Landon MB, Mele L, Spong CY, et al. The relationship between maternal glycemia and perinatal outcome. Obstet Gynecol 2011; 117 (2 Pt 1): 218-24.

54. Makgoba M, Savvidou MD, Steer PJ. An analysis of the interrelationship between maternal age, body mass index and racial origin in the development of gestational diabetes mellitus. BJOG 2012; 119 (3): 276-82.

55. Tobias DK, Zhang C, van Dam RM, Bowers K, Hu FB. Physical activity before and during pregnancy and risk of gestational diabetes mellitus: a meta-analysis. Diabetes Care 2011; 34 (1): 223-9.

56. Hanson MA, Low FM, Gluckman PD. Epigenetic Epidemiology: The Rebirth of Soft Inheritance. Ann Nutr Metab 2011; 58 (Suppl. 2): 8-15.

57. Hanson MA, Gluckman PD. Developmental origins of health and disease: Moving from biological concepts to interventions and policy. Int J Gynaecol Obstet 2011; 115 (Suppl 1): S3-S5.

58. Dabelea D, Crume T. Maternal environment and the transgenerational cycle of obesity and diabetes. Diabetes 2011; 60 (7): 1849-55.

59. Catalano PM, McIntyre HD, Cruickshank JK, et al. The Hyperglycemia and Adverse Pregnancy Outcome Study: Associations of GDM and obesity with pregnancy outcomes. Diabetes Care 2012; 35 (4): 780-6.

60. Catalano PM. Obesity and pregnancy - the propagation of a viscous cycle? J Clin Endocrinol Metab $2003 ; \mathbf{8 8}$ (8): 3505-6.

61. Gillman MW, Oakey H, Baghurst PA, Volkmer RE, Robinson JS, Crowther CA. Effect of treatment of gestational diabetes mellitus on obesity in the next generation. Diabetes Care 2010; 33 (5): 964-8.

62. Beaglehole R, Bonita R, Horton R, et al. Priority actions for the non-communicable disease crisis. Lancet 2011; 377 (9775): 1438-47. 